\title{
Intriguingly Simple and Fast Transit Routing*
}

\author{
Julian Dibbelt, Thomas Pajor, Ben Strasser, and Dorothea Wagner \\ Karlsruhe Institute of Technology (KIT), 76128 Karlsruhe, Germany. \\ \{dibbelt, pajor, strasser, dorothea.wagner\}@kit.edu
}

\begin{abstract}
This paper studies the problem of computing optimal journeys in dynamic public transit networks. We introduce a novel algorithmic framework, called Connection Scan Algorithm (CSA), to compute journeys. It organizes data as a single array of connections, which it scans once per query. Despite its simplicity, our algorithm is very versatile. We use it to solve earliest arrival and multi-criteria profile queries. Moreover, we extend it to handle the minimum expected arrival time (MEAT) problem, which incorporates stochastic delays on the vehicles and asks for a set of (alternative) journeys that in its entirety minimizes the user's expected arrival time at the destination. Our experiments on the dense metropolitan network of London show that CSA computes MEAT queries, our most complex scenario, in $272 \mathrm{~ms}$ on average.
\end{abstract}

\section{Introduction}

Commercial public transit route planning systems are confronted with millions of queries per hour [12], making fast algorithms a necessity. Preprocessing-based techniques for computing point-to-point shortest paths have been very successful on road networks $[8,16]$, but their adaption to public transit networks $[2,10]$ is harder than expected $[1,3,4]$. The problem of computing "best" journeys comes in several variants [14]: The simplest, called earliest arrival, takes a departure time as input, and determines a journey that arrives at the destination as early as possible. If further criteria, such as the number of transfers, are important, one may consider multi-criteria optimization $[7,9]$. Finally, a profile query $[6,7]$ computes a set of optimal journeys that depart during a period of time (such as a day). Traditionally, these problems have been solved by (variants of) Dijkstra's algorithm on an appropriate graph model. Well-known examples are the timeexpanded and time-dependent models $[6,10,14,15]$. Recently, Delling et al. [7] introduced RAPTOR. It solves the multi-criteria problem (arrival time and number of transfers) by using dynamic programming directly on the timetable, hence, no longer requires a graph or a priority queue.

In this work, we present the Connection Scan Algorithm (CSA). In its basic variant, it solves the earliest arrival problem, and is, like RAPTOR, not graph-based. However, it is not centered around routes (as RAPTOR), but elementary connections, which are the most basic building block of a timetable.

\footnotetext{
* Partial support by DFG grant WA654/16-1 and EU grant 288094 (eCOMPASS).
} 
CSA organizes them as one single array, which it then scans once (linearly) to compute journeys to all stops of the network. The algorithm turns out to be intriguingly simple with excellent spatial data locality. We also extend CSA to handle multi-criteria profile queries: For a full time period, it computes Pareto sets of journeys optimizing arrival time and number of transfers. Finally, we introduce the minimum expected arrival time problem (MEAT). It incorporates uncertainty $[5,9,11]$ by considering stochastic delays on the vehicles. Its goal is to compute a set of journeys that minimizes the user's $e x$ pected arrival time (at the destination). The output can be viewed as a decision graph that provides all relevant alternative journeys at stops where transfers might fail (see Fig. 1). We extend CSA to handle these queries very efficiently. Moreover, we do not make use of heavy preprocessing, thus, enabling dynamic

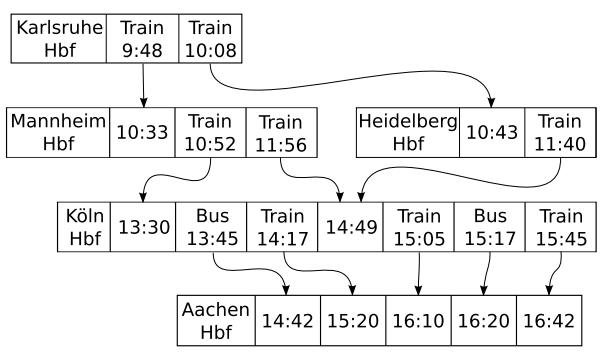

Fig. 1. Delay-robust itinerary from Karlsruhe to Aachen, Germany. A user should try to take the leftmost path. If transfers fail, alternatives are available. scenarios including train cancellations, route changes, real-time delays, etc. Our experiments on the dense metropolitan network of London validate our approach. With CSA, we compute earliest arrival queries in under $2 \mathrm{~ms}$, and multi-criteria profile queries for a full period in $221 \mathrm{~ms}$ - faster than previous algorithms. Moreover, we solve the most complex of our problems, MEAT, with CSA in $272 \mathrm{~ms}$, fast enough for interactive applications.

This paper is organized as follows. Section 2 sets necessary notion, and Section 3 presents our new algorithm. Section 4 extends it to multi-criteria profile queries, while Section 5 considers MEAT. The experimental evaluation is available in Section 6, while Section 7 contains concluding remarks.

\section{Preliminaries}

Our public transit networks are defined in terms of their aperiodic timetable, consisting of a set of stops, a set of connections, and a set of footpaths. A stop $p$ corresponds to a location in the network where a passenger can enter or exit a vehicle (such as a bus stop or train station). Stops may have associated minimum change times, denoted $\tau_{\mathrm{ch}}(p)$, which represent the minimum time required to change vehicles at $p$. A connection $c$ models a vehicle departing at a stop $p_{\text {dep }}(c)$ at time $\tau_{\text {dep }}(c)$ and arriving at stop $p_{\text {arr }}(c)$ at time $\tau_{\text {arr }}(c)$ without intermediate halt. Connections that are subsequently operated by the same vehicle are grouped into trips. We identify them by $t(c)$. We denote by $c_{\text {next }}$ the next connection (after $c$ ) of the same trip, if available. Trips can be further grouped into routes. A route is a set of trips serving the exact same sequence of stops. For correctness, we require trips of the same route to not overtake each other. Footpaths enable walking transfers between nearby stops. Each footpath consists of two stops 
with an associated walking duration. Note that our footpaths are transitively closed. A journey is a sequence of connections and footpaths. If two subsequent connections are not part of the same trip, their arrival-departure time-difference must be at least the minimum change time of the stop. Because our footpaths are transitively closed, a journey never contains two subsequent footpaths.

In this paper we consider several well-known problems. In the earliest arrival problem we are given a source stop $p_{s}$, a target stop $p_{t}$, and a departure time $\tau$. It asks for a journey that departs from $p_{s}$ no earlier than $\tau$ and arrives at $p_{t}$ as early as possible. The profile problem asks for the set of all earliest arrival journeys (from $p_{s}$ to $p_{t}$ ) for every departure at $p_{s}$. Besides arrival time, we also consider the number of transfers as criterion: In multi-criteria scenarios one is interested in computing a Pareto set of nondominated journeys. Here, a journey $J_{1}$ dominates a journey $J_{2}$ if it is better with respect to every criterion. Nondominated journeys are also called to be Pareto-optimal. Finally, the multicriteria profile problem requests a set of Pareto-optimal journeys (from $p_{s}$ to $p_{t}$ ) for all departures (at $p_{s}$ ).

Usually, these problems have been solved by (variants of) Dijkstra's algorithm on an appropriate graph (representing the timetable). Most relevant to our work is the realistic time-expanded model [15]. It expands time in the sense that it creates a vertex for each event in the timetable (such as a vehicle departing or arriving at a stop). Then, for every connection it inserts an arc between its respective departure/arrival events, and also arcs that link subsequent connections. Arcs are always weighted by the time difference of their linked events. Special vertices may be added to respect minimum change times at stops. See [14, 15] for details.

\section{Basic Connection Scan Algorithm}

We now introduce the Connection Scan Algorithm (CSA), our approach to public transit route planning. We describe it for the earliest arrival problem and extend it to more complex scenarios in Sections 4 and 5. Our algorithm builds on the following property of public transit networks: We call a connection c reachable iff either the user is already traveling on a preceding connection of the same trip $t(c)$, or, he is standing at the connection's departure stop $p_{\text {dep }}(c)$ on time, i.e., before $\tau_{\text {dep }}(c)$. In fact, the time-expanded approach encodes this property into a graph $G$, and then uses Dijkstra's algorithm to obtain optimal sequences of reachable connections [15]. Unfortunately, Dijkstra's performance is affected by many priority queue operations and suboptimal memory access patterns. However, since our timetables are aperiodic, we observe that $G$ is acyclic. Thus, its arcs may be sorted topologically, e. g., by departure time. Dijkstra's algorithm on $G$, actually, scans (a subsequence of) them in this order.

Instead of building a graph, our algorithm assembles the timetable's connections into a single array $C$, sorted by departure time. Given source stop $p_{s}$ and departure time $\tau$ as input, it maintains for each stop $p$ a label $\tau(p)$ representing the earliest arrival time at $p$. Labels $\tau(\cdot)$ are initialized to all-infinity, except $\tau\left(p_{s}\right)$, which is set to $\tau$. The algorithm scans all connections $c \in C$ (in order), testing 
if $c$ can be reached. If this is the case and if $\tau_{\text {arr }}(c)$ improves $\tau\left(p_{\text {arr }}(c)\right)$, CSA relaxes $c$ by updating $\tau\left(p_{\text {arr }}(c)\right)$. After scanning the full array, the labels $\tau(\cdot)$ provably hold earliest arrival times for all stops.

Reachability, Minimum Change Times and Footpaths. To account for minimum change times in our data, we check a connection $c$ for reachability by testing if $\tau\left(p_{\text {dep }}(c)\right)+\tau_{\text {ch }}\left(p_{\text {dep }}(c)\right) \leq \tau_{\text {dep }}(c)$ holds. Additionally, we track whether a preceding connection of the same trip $t(c)$ has been used. We, therefore, maintain for each connection a flag, initially set to 0 . Whenever the algorithm identifies a connection $c$ as reachable, it sets the flag of $c$ 's subsequent connection $c_{\text {next }}$ to 1 . Note that for networks with $\tau_{\mathrm{ch}}(\cdot)=0$, trip tracking can be disabled and testing reachability simplifies to $\tau\left(p_{\text {dep }}(c)\right) \leq \tau_{\text {dep }}(c)$. To handle footpaths, each time the algorithm relaxes a connection $c$, it scans all outgoing footpaths of $p_{\text {arr }}(c)$.

Improvements. Clearly, connections departing before time $\tau$ can never be reached and need not be scanned. We do a binary search on $C$ to identify the first relevant connection and start scanning from there (start criterion). If we are only interested in one-to-one queries, the algorithm may stop as soon as it scans a connection whose departure time exceeds the target stop's earliest arrival time. Also, as soon as one connection of a trip is reachable, so are all subsequent connections of the same trip (and preceding connections of the trip have already been scanned). We may, therefore, keep a flag (indicating reachability) per trip (instead of per connection). The algorithm then operates on these trip flags instead. Note that we store all data sequentially in memory, making the scan extremely cache-efficient. Only accesses to stop labels and trip flags are potentially costly, but the number of stops and trips is small in comparison. To further improve spatial locality, we subtract from each connection $c \in C$ the minimum change time of $p_{\text {dep }}(c)$ from $\tau_{\text {dep }}(c)$, but keep the original ordering of $C$. Hence, CSA requires random access only on small parts of its data, which mostly fits in low-level cache.

\section{Extensions}

CSA can be extended to profile queries. Given the timetable and a source stop $p_{s}$, a profile query computes for every stop $p$ the set of all earliest arrival journeys to $p$ for every departure from $p_{s}$, discarding dominated journeys. Such queries are useful for preprocessing techniques, but also for users with flexible departure (or arrival) time. We refer to the solution as a Pareto set of $\left(\tau_{\text {dep }}\left(p_{s}\right), \tau_{\text {arr }}\left(p_{t}\right)\right)$ pairs.

In the following, we describe the reverse $p-p_{t}$-profile query, which is needed in Section 5. The forward search works analogously. Our algorithm, pCSA (p for profile), scans once over the array of connections sorted by decreasing departure time. For every stop it keeps a partial (tentative) profile. It maintains the property that the partial profiles are correct wrt. the subset of already scanned connections. Every stop is initialized with an empty profile, except $p_{t}$, which is set to a constant identity-profile. When scanning a connection $c$, pCSA evaluates the partial profile at the arrival stop $p_{\text {arr }}(c)$ : It asks for the earliest arrival time $\tau^{*}$ at $p_{t}$ over 
all journeys departing at $p_{\text {arr }}(c)$ at $\tau_{\text {arr }}(c)$ or later. It then updates the profile at $p_{\text {dep }}(c)$ by potentially adding the pair $\left(\tau_{\text {dep }}(c), \tau^{*}\right)$ to it, discarding newly dominated pairs, if necessary.

Maintaining Profiles. We describe two variants of maintaining profiles. The first, pCSA-P (P for Pareto), stores them as arrays of Pareto-optimal $\left(\tau_{\text {dep }}, \tau_{\text {arr }}\right)$ pairs ordered by decreasing arrival (departure) time. Since new candidate entries are generated in order of decreasing departure time, profile updates are a constanttime operation: A candidate entry is either dominated by the last entry or is appended to the array. Profile evaluation is implemented as a linear scan over the array. This is quick in practice, since, compared to the timetable's period, connections usually have a short duration. The identity profile of $p_{t}$ is handled as a special case. By slightly modifying the data structure, we obtain pCSA-C (C for constant), for which evaluation is also possible in constant time: When updating a profile, pCSA may append a candidate entry, even if it is dominated. To ensure correctness, we set the candidate's arrival time $\tau^{*}$ to that of the dominating entry. We then observe that, independent of the input's source or target stop, profile entries are always generated in the same order. Moreover, each connection is associated with only two such entries, one at its departure stop, relevant for updating, and, one at its arrival stop, relevant for evaluation. For each connection, we precompute profile indices pointing to these two entries, keeping them with the connection. Furthermore, its associated departure time and stop may be dropped. Note that the space consumption for keeping all (even suboptimal) profile entries is bounded by the number of connections. Following [6], we also collect - in a quick preprocessing step - at each stop all arrival times (in decreasing order). Then, instead of storing arrival times in the profile entries, we keep arrival time indices. For our scenarios, these can be encoded using 16 (or fewer) bits. We call this technique time indexing, and the corresponding algorithm pCSA-CT.

Minimum Change Times and Footpaths. We incorporate minimum change times by evaluating the profile at a stop $p$ for time $\tau$ at $\tau+\tau_{\operatorname{ch}}(p)$. The trip bit is replaced by a trip arrival time, which represents the earliest arrival time at $p_{t}$ when continuing with the trip. When scanning a connection $c$, we take the minimum of the trip arrival time and the evaluated profile at $p_{\text {arr }}(c)$. We update the trip arrival time and the profile at $p_{\text {dep }}(c)$, accordingly. Footpaths are handled as follows. Whenever a connection $c$ is relaxed, we scan all incoming footpaths at $p_{\text {dep }}(c)$. However, this no longer guarantees that profile entries are generated by decreasing departure time, making profile updates a non-constant operation for pCSA-P. Also, we can no longer precompute profile indices for pCSA-C. Therefore, we expand footpaths into pseudoconnections in our data, as follows. If $p_{a}$ and $p_{b}$ are connected by a footpath, we look at all reachable (via the footpath) pairs of incoming connections $c_{\text {in }}$ at $p_{a}$ and outgoing connections $c_{\text {out }}$ at $p_{b}$. We create a new pseudoconnection (from $p_{a}$ to $p_{b}$, departure time $\tau_{\text {arr }}\left(c_{\text {in }}\right.$ ), and arrival time $\left.\tau_{\text {dep }}\left(c_{\text {out }}\right)\right)$ iff there is no other pseudoconnection with a later or equal departure time and an earlier or equal arrival time. Pseudoconnections can be identified by a simultaneous sweep over the incoming/outgoing connections 
of $p_{a}$ and $p_{b}$. During query, we handle footpaths toward $p_{t}$ as a special case of the evaluation procedure. Footpaths at $p_{s}$ are handled by merging the profiles of stops that are reachable by foot from $p_{s}$.

One-to-One Queries. So far we described all-to-one profile queries, i. e., from all stops to the target stop $p_{t}$. If only the one-to-one profile between stops $p_{s}$ and $p_{t}$ is of interest, a well-known pruning rule $[6,14]$ can be applied to pCSA-P: Before inserting a new profile entry at any stop, we check whether it is dominated by the last entry in the profile at $p_{s}$. If so, the current connection cannot possibly be extended to a Pareto-optimal solution at the source, and, hence, can be pruned. However, we still have to continue scanning the full connection array.

Multi-Criteria. CSA can be extended to compute multi-criteria profiles, optimizing triples $\left(\tau_{\text {dep }}\left(p_{s}\right), \tau_{\text {arr }}\left(p_{t}\right), \# t\right)$ of departure time, arrival time and number of taken trips. We call this variant mcpCSA-CT. We organize these triples hierarchically by mapping arrival time $\tau_{\text {arr }}\left(p_{t}\right)$ onto bags of $\left(\tau_{\text {dep }}\left(p_{s}\right)\right.$, \#t) pairs. Thus, we follow the general approach of pCSA-CT, but now maintain profiles as $\left(\tau_{\text {arr }}\left(p_{t}\right)\right.$, bag $)$ pairs. Evaluating a profile, thus, returns a bag. Where pCSA-CT computes the minimum of two departure times, mcpCSA-CT merges two bags, i. e., it computes their union and removes dominated entries. When it scans a connection $c$, \#t is increased by one for each entry of the evaluated bag, unless $c$ is a pseudoconncetion. It then merges the result with the bag of trip $t(c)$, and updates the profile at $p_{\text {dep }}(c)$, accordingly. Exploiting that, in practice, $\# t$ only takes small integral values, we store bags as fixed-length vectors using \# $t$ as index and departure times as values. Merging bags then corresponds to a componentwise minimum, and increasing \#t to shifting the vector's values. A variant, mcpCSA-CT-SSE, uses SIMD-instructions for these operations.

\section{Minimum Expected Arrival Time}

In this section we aim to provide delay-robust journeys that offer sensible backup alternatives at every stop for the case that transfers fail. A tempting approach might be to optimize reliability, introduced in [9], possibly together with other criteria. While this produces journeys that have low failure probabilities on their transfers, they are not necessarily robust in our sense: The set of reliable journeys may already diverge at the source stop, and in general, no fall-back alternatives are guaranteed at intermediate stops. On the other hand, on high-frequency urban routes (such as subways) an unreliable transfer might not be a problem, if the next feasible trip is just a few minutes away. To ensure that the user is never left without guidance, we compute a subset of connections (rather than journeys) such that at any point along the way, the user is provided with a good (in terms of arrival time) option for continuing his journey toward the destination. We propose to minimize the expected arrival time to achieve these goals.

We assume the following simple delay model: A connection $c$ arrives at a random time $\tau_{\text {arr }}^{R}(c)$ but departs on time at $\tau_{\text {dep }}(c)$. All random arrival times are 
independent. No connection arrives earlier than its scheduled arrival time $\tau_{\text {arr }}(c)$. To make computations meaningful, we assume an upper bound on all $\tau_{\text {arr }}^{R}(c)$. We further assume that walking is exact. Note that more complex stochastic models have been considered in $[5,11]$, containing dependent random variables to model delays. In this case, however, such models also propagate data errors (besides delays), therefore, requiring precise delay data [5], which is hard to obtain in practice. Also, even basic operations in [11] have super-quadratic running time (in the number of connections), making the approach impractical, already for medium-sized timetables.

For a given target stop $p_{t}$, we define for every subset $S$ of connections of the timetable and for every connection $c$ the expected arrival time $\hat{\tau}(S, c)$ at $p_{t}$, recursively. Let $c_{1} \ldots c_{n} \subseteq S$ be the connections that the user can transfer to at $c$ 's arrival stop $p_{\text {arr }}(c)$, ordered by departure time $\tau_{\text {dep }}\left(c_{i}\right)$ (adjusted for footpaths and minimum change times). We define

$$
\hat{\tau}(S, c)=\min \left\{\hat{\tau}\left(S, c_{\text {next }}\right), \sum_{i=1}^{n+1} P\left[\tau_{\text {dep }}\left(c_{i-1}\right) \leq \tau_{\text {arr }}^{R}(c)<\tau_{\text {dep }}\left(c_{i}\right)\right] \cdot \hat{\tau}\left(S, c_{i}\right)\right\}
$$

where $\tau_{\text {dep }}\left(c_{0}\right)=\tau_{\text {arr }}(c), \tau_{\text {dep }}\left(c_{n+1}\right)=\infty, \hat{\tau}\left(S, c_{n+1}\right)=\infty$, and $\hat{\tau}\left(S, c_{\text {next }}\right)=\infty$ if $c$ is the last connection of trip $t(c)$. The base of the recursion is defined by the connections $c$ arriving at $p_{t}$, for which we define $\hat{\tau}(S, c)=E\left[\tau_{\text {arr }}^{R}(c)\right]$. If the possibility of the user not reaching the target is non-zero, the expected arrival time is trivially $\infty$. Since a connection is assumed to never arrive early, $\hat{\tau}(S, c)$ only depends on connections departing later than $c$, which guarantees termination. (This is where we require aperiodicity; in periodic networks infinite recursions may occur.) In short, we compute the average over the expected arrival times of each outgoing connection from the stop $p_{\text {arr }}(c)$, weighted by the probability of the user catching it. We define the minimum expected arrival time $\hat{\tau}^{*}(c)$ of a connection $c$ as the minimum $\hat{\tau}(S, c)$ over all subsets $S$. A subset $S^{*}$ minimizes $\hat{\tau}^{*}(c)$, if for every stop $p$ the set of pair $\left(\tau_{\text {dep }}(c), \hat{\tau}\left(S^{*}, c\right)\right)$ induced by those $c \in S^{*}$ that depart at $p$, does not include dominated connections. (A pair is dominated, if, wrt. another pair, it departs earlier with higher expected arrival time.) Note that removing a dominated pair's connection improves $\hat{\tau}(\cdot)$. Also, all subsets with this property have the same $\hat{\tau}(\cdot)$ and therefore $S^{*}$ is globally optimal. At least one subset $S^{*}$ exists that is optimal for every $c$, because removing dominated connections is independent of $c$.

To solve the minimum expected arrival time problem (MEAT), we compute a set $S^{*}$, and output the reachable connections for the desired source stop and departure time. Our algorithm is based directly on pCSA-P, with a different meaning for its stop labels: Instead of mapping a departure time $\tau_{\text {dep }}$ to the corresponding earliest arrival time $\tau_{\text {arr }}$ at $p_{t}$, the algorithm now maps $\tau_{\text {dep }}$ to the corresponding minimum expected arrival time $\hat{\tau}^{*}$ at $p_{t}$. It does so by maintaining an array of nondominated $\left(\tau_{\text {dep }}, \hat{\tau}^{*}\right)$ pairs. For a connection $c$, the label at stop $p_{\text {arr }}(c)$ is evaluated by a linear scan over that array: Following from the recursive definition above, the minimum expected arrival time $\hat{\tau}^{*}(c)$ is computed by a weighted summation of each of the expected arrival times $\hat{\tau}^{*}$ collected during 
Table 1. Size figures for our timetables including figures of the time-dependent (TD), colored time-dependent (TD-col), and time-expanded (TE) graph models $[6,14,15]$.

\begin{tabular}{|c|c|c|c|c|c|c|}
\hline Figures & & London & & Germany & & Europe \\
\hline Stops & & 20843 & & 6822 & & 30517 \\
\hline Trips & & 125537 & & 94858 & & 463887 \\
\hline Connections & & 4850431 & & 976678 & & 4654812 \\
\hline Routes & & 2135 & & 9055 & & 42547 \\
\hline Footpaths & & 45652 & & 0 & & 0 \\
\hline Expanded Footpaths & & 8436763 & & 0 & & 0 \\
\hline TD Vertices (Arcs) & $97 \mathrm{k}$ & $(272 \mathrm{k})$ & $114 \mathrm{k}$ & $(314 \mathrm{k})$ & $527 \mathrm{k}$ & $(1448 \mathrm{k})$ \\
\hline TD-col Vertices (Arcs) & $21 \mathrm{k}$ & $(71 \mathrm{k})$ & $20 \mathrm{k}$ & $(86 \mathrm{k})$ & $79 \mathrm{k}$ & $(339 \mathrm{k})$ \\
\hline TE Vertices (Arcs) & $9338 \mathrm{k}$ & $(34990 \mathrm{k})$ & $1809 \mathrm{k}$ & $(3652 \mathrm{k})$ & $8778 \mathrm{k}$ & $(17557 \mathrm{k})$ \\
\hline
\end{tabular}

this scan multiplied with the success probability of the corresponding transfer at $p_{\text {arr }}(c)$. An optimization, called earliest arrival pruning, first runs an earliest arrival query from the source stop and then only processes connections marked reachable during that query. Note that, since during evaluation we scan over several outgoing connections, pCSA-C is not applicable.

\section{Experiments}

We ran experiments pinned to one core of a dual 8-core Intel Xeon E5-2670 clocked at $2.6 \mathrm{GHz}$, with $64 \mathrm{GiB}$ of DDR3-1600 RAM, $20 \mathrm{MiB}$ of L3 and $256 \mathrm{KiB}$ of L2 cache. We compiled our $\mathrm{C}++$ code using $\mathrm{g}++4.7 .1$ with flags -03 -mavx.

We consider three realistic inputs whose sizes are reported in Table 1. They are also used in $[6,10,7]$, but we additionally filter them for (obvious) errors, such as duplicated trips and connections with non-positive travel time. Our main instance, London, is available at [13]. It includes tube (subway), bus, tram, Dockland Light Rail (DLR) and is our only instance that also includes footpaths. However, it has no minimum change times. The German and European networks were kindly provided by HaCon [12]. Both have minimum change times. The German network contains long-distance, regional, and commuter trains operated by Deutsche Bahn during the winter schedule of 2001/02. The European network contains long-distance trains, and is based on the winter schedule of 1996/97. To account for overnight trains and long journeys, our (aperiodic) timetables cover one (London), two (Germany), and three (Europe) consecutive days.

We ran for every experiment 10000 queries with source and target stops chosen uniformly at random. Departure times are chosen at random between 0:00 and 24:00 (of the first day). We report the running time and the number of label comparisons, counting an SSE operation as a single comparison. Note that we disregard comparisons in the priority queue implementation.

Earliest Arrival. In Table 2, we report performance figures for several algorithms on the London instance. Besides CSA, we ran realistic time-expanded 
Table 2. Figures for the earliest arrival problem on our London instance. Indicators are: - enabled, o disabled, - not applicable. "Sta." refers to the start criterion. "Trp." indicates the method of trip tracking: connection flag (o), trip flag $(\bullet)$, none $(\times)$. "One." indicates one-to-one queries by either using the stop criterion or pruning.

\begin{tabular}{|c|c|c|c|c|c|c|c|c|}
\hline Alg. & के & & $\begin{array}{l}\text { \# Scanned } \\
\text { Arcs/Con. }\end{array}$ & $\begin{array}{c}\text { \# Reachable } \\
\text { Arcs/Con. }\end{array}$ & $\begin{array}{l}\text { \# Relaxed } \\
\text { Arcs/Con. }\end{array}$ & $\begin{array}{r}\text { \# Scanned } \\
\text { Footpaths }\end{array}$ & $\begin{array}{r}\text { \# L.Cmp. } \\
\text { p. Stop }\end{array}$ & $\begin{array}{r}\text { Time } \\
{[\mathrm{ms}]}\end{array}$ \\
\hline $\mathrm{TE}$ & $-\quad-$ & ० & 20370117 & - & 5739046 & - & 977.3 & 876.2 \\
\hline TD & $-\quad-$ & $\circ$ & 262080 & - & 115588 & - & 11.9 & 18.9 \\
\hline TD-col & $-\quad-$ & ० & 68183 & - & 21294 & - & 3.2 & 7.3 \\
\hline CSA & $\circ 0$ & $\circ$ & 4850431 & 2576355 & 11090 & 11500 & 356.9 & 16.8 \\
\hline CSA & - 0 & ० & 2908731 & 2576355 & 11090 & 11500 & 279.7 & 12.4 \\
\hline CSA & $\bullet \bullet$ & $\circ$ & 2908731 & 2576355 & 11090 & 11500 & 279.7 & 9.7 \\
\hline $\mathrm{TE}$ & $-\quad-$ & $\bullet$ & 1391761 & - & 385641 & - & 66.8 & 64.4 \\
\hline $\mathrm{TD}$ & $-\quad-$ & $\bullet$ & 158840 & - & 68038 & - & 7.2 & 10.9 \\
\hline TD-col & $-\quad-$ & $\bullet$ & 43238 & - & 11602 & - & 2.1 & 4.1 \\
\hline CSA & $\bullet \bullet$ & $\bullet$ & 420263 & 126983 & 5574 & 7005 & 26.6 & 2.0 \\
\hline CSA & - $\times$ & $\bullet$ & 420263 & 126983 & 5574 & 7005 & 26.6 & 1.8 \\
\hline
\end{tabular}

Dijkstra (TE) with two vertices per connection [15] and footpaths [14], realistic time-dependent Dijkstra (TD), and time-dependent Dijkstra using the optimized coloring model [6] (TD-col). For CSA, we distinguish between scanned, reachable and relaxed connections. Algorithms in Table 2 are grouped into blocks.

The first considers one-to-all queries, and we see that basic CSA scans all connections $(4.8 \mathrm{M})$, only half of which are reachable. On the other hand, TE scans about half of the graph's arcs $(20 \mathrm{M})$. Still, this is a factor of four more entities due to the modeling overhead of the time-expanded graph. Regarding query time, CSA greatly benefits from its simple data structures and lack of priority queue: It is a factor of 52 faster than TE. Enabling the start criterion reduces the number of scanned connections by $40 \%$, which also helps query time. Using trip bits increases spatial locality and further reduces query time to $9.7 \mathrm{~ms}$. We observe that just a small fraction of scanned arcs/connections actually improve stop labels. Only then CSA must consider footpaths. The second block considers one-to-one queries. Here, the number of connections scanned by CSA is significantly smaller; journeys in London rarely have long travel times. Since our London instance does not have minimum change times, we may remove trip tracking from the algorithm entirely. This yields the best query time of $1.8 \mathrm{~ms}$ on average. Although CSA compares significantly more labels, it outperforms Dijkstra in almost all cases (also see Table 4 for other inputs). Only for one-to-all queries on London TD-col is slightly faster than CSA.

Profile and Multi-Criteria Queries. In Table 3 we report experiments for (multicriteria) profile queries on London. Other instances are available in Table 4. We compare CSA to SPCS-col [6] (an extension of TD-col to profile queries) and rRAPTOR [7] (an extension of RAPTOR to multi-criteria profile queries). 
Table 3. Figures for the (multi-criteria) profile problem on London. "\# Tr." is the max. number of trips considered. "Arr." indicates minimizing arrival time, "Tran." transfers. "Prof." indicates profile queries. "\# Jn." is the number of Pareto-optimal journeys.

\begin{tabular}{|c|c|c|c|c|c|c|}
\hline Algorithm & $\hat{x}$ & Fं & & 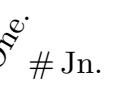 & $\begin{array}{l}\text { \# L.Cmp. } \\
\text { p. Stop }\end{array}$ & $\begin{array}{r}\text { Time } \\
{[\mathrm{ms}]}\end{array}$ \\
\hline SPCS-col & - & - 0 & - 0 & 98.2 & 477.7 & 1262 \\
\hline SPCS-col & - & - 0 & - • & 98.2 & 372.5 & 843 \\
\hline pCSA-P & - & - 0 & - 0 & 98.2 & 567.6 & 177 \\
\hline pCSA-P & - & - 0 & - • & 98.2 & 436.9 & 161 \\
\hline $\mathrm{pCSA}-\mathrm{C}$ & - & - 0 & - - & 98.2 & 1912.5 & 134 \\
\hline pCSA-CT & - & - $\circ$ & - - & 98.2 & 1912.5 & 104 \\
\hline rRAPTOR & 8 & $\bullet$ & - & 203.4 & 1812.5 & 1179 \\
\hline rRAPTOR & 8 & $\bullet$ & $\bullet \bullet$ & 203.4 & 1579.6 & 878 \\
\hline rRAPTOR & 16 & $\bullet$ & - • & 206.4 & 1634.0 & 922 \\
\hline mcpCSA-CT & 8 & $\bullet \bullet$ & - - & $-\quad 203.4$ & 15299.8 & 255 \\
\hline mcpCSA-CT-SSE & 8 & $\bullet \bullet$ & - - & $-\quad 203.4$ & 1912.5 & 221 \\
\hline mcpCSA-CT-SSE & 16 & $\bullet \bullet$ & - - & $-\quad 206.4$ & 3824.9 & 466 \\
\hline
\end{tabular}

Note that in [7] rRAPTOR is evaluated on two-hours range queries, whereas we compute full profile queries. A first observation is that, regarding query time, one-to-all SPCS is outperformed by all other algorithms, even those which additionally minimize the number of transfers. Similarly to our previous experiment, CSA generally does more work than the competing algorithms, but is, again, faster due to its cache-friendlier memory access patterns. We also observe that one-to-all pCSA-C is slightly faster than pCSA-P, even with target pruning enabled, although it scans 2.7 times as many connections because of expanded footpaths. Note, however, that the figure for pCSA-C does not include the postprocessing that removes dominated journeys. Time indexing further accelerates pCSA-C, indicating that the algorithm is, indeed, memory-bound. Regarding multi-criteria profile queries, doubling the number of considered trips also doubles both CSA's label comparisons and its running time. For rRAPTOR the difference is less (only 12\%) - most work is spent in the first eight rounds. Indeed, journeys with more than eight trips are very rare. This justifies mcpCSA-CT-SSE with eight trips, which is our fastest algorithm (221 ms on average). Note that using an AVX2 processor (announced for June 2013), one will be able to process 256 bitvectors in a single instruction. We, therefore, expect mcpCSA-CT-SSE to perform better for greater numbers of trips in the future.

Minimum Expected Arrival Time. In Table 5 we present figures for the MEAT problem on all instances. Besides running time, we also report output complexity in terms of number of stops and arcs of the decision graph (see Fig. 1 for an example). Real world delay data was not available to us. Hence, we follow Disser et al. [9] and assume that the probability of a train being delayed by $t$ minutes (or less) is $0.99-0.4 \cdot \exp (-t / 8)$. After $30 \mathrm{~min}(10 \mathrm{~min}$ on London) we 
Table 4. Evaluating other instances. Start criterion and trip flags are always used.

\begin{tabular}{|c|c|c|c|c|c|c|c|c|c|c|}
\hline Algorithm & \multicolumn{4}{|c|}{ से } & $\begin{array}{r}\text { \# } \\
\text { \# Jn. }\end{array}$ & $\begin{array}{r}\text { Germ } \\
\text { t L.Cmp. } \\
\text { p. Stop }\end{array}$ & $\begin{array}{l}\text { Timy } \\
\text { Tims] }\end{array}$ & $\begin{array}{l}\text { \# } \\
\text { \# Jn. }\end{array}$ & $\begin{array}{l}\text { Europe } \\
\text { L.Cmp. } \\
\text { p. Stop }\end{array}$ & $\begin{array}{r}\text { Time } \\
{[\mathrm{ms}]}\end{array}$ \\
\hline $\mathrm{TE}$ & - & - 0 & $\circ$ & $\circ$ & 1.0 & 317.0 & 117.1 & 0.9 & 288.6 & 624.1 \\
\hline TD-col & - & - 0 & ० & $\circ$ & 1.0 & 11.9 & 3.5 & 0.9 & 10.0 & 21.6 \\
\hline CSA & - & - 0 & . & $\circ$ & 1.0 & 228.7 & 3.4 & 0.9 & 209.5 & 19.5 \\
\hline $\mathrm{TE}$ & - & - 0 & $\circ$ & $\bullet$ & 1.0 & 29.8 & 11.7 & 0.9 & 56.3 & 129.9 \\
\hline TD-col & - & - 0 & $\circ$ & $\bullet$ & 1.0 & 6.8 & 2.0 & 0.9 & 5.3 & 11.5 \\
\hline CSA & - & - 0 & 0 & $\bullet$ & 1.0 & 40.8 & 0.8 & 0.9 & 74.2 & 8.3 \\
\hline pCSA-CT & - & - 0 & $\bullet$ & - & 20.2 & 429.5 & 4.9 & 11.4 & 457.6 & 46.2 \\
\hline rRAPTOR & 8 & - • & $\bullet$ & $\circ$ & 29.4 & 752.1 & 161.3 & 17.2 & 377.5 & 421.8 \\
\hline rRAPTOR & 8 & - • & $\bullet$ & $\bullet$ & 29.4 & 640.1 & 123.0 & 17.2 & 340.8 & 344.9 \\
\hline mcpCSA-CT-SSE & 8 & - • & $\bullet$ & - & 29.4 & 429.5 & 17.9 & 17.2 & 457.6 & 98.2 \\
\hline
\end{tabular}

Table 5. Evaluating pCSA-P for the MEAT problem on all instances.

\begin{tabular}{|c|c|c|c|c|c|c|}
\hline Network & $\begin{array}{r}\text { Max. Delay } \\
{[\min ]}\end{array}$ & $\begin{array}{l}\text { Decision } \\
\text { \# Stops }\end{array}$ & $\begin{array}{l}\text { Graph } \\
\text { \# Arcs }\end{array}$ & $\begin{array}{r}\text { All-To-One } \\
\text { Time }[\mathrm{ms}]\end{array}$ & $\begin{array}{r}\text { One-To-One } \\
\text { Time }[\mathrm{ms}]\end{array}$ & $\begin{array}{r}\text { One-To-One } \\
\text { Dis. Time }[\mathrm{ms}]\end{array}$ \\
\hline Germany & 30 & 8 & 19 & 68.1 & 31.0 & 24.6 \\
\hline Europe & 30 & 20 & 46 & 205.0 & 169.0 & 112.0 \\
\hline London & 10 & 2724 & 30243 & 668.0 & 491.0 & 272.0 \\
\hline
\end{tabular}

set this value to 1 . Moreover, we also evaluate performance when discretizing the probability function at 60 equidistant points [9]. We run pCSA-P on 10000 random queries and evaluate both the all-to-one and one-to-one (with earliest arrival pruning enabled) setting. Regarding output complexity, on the German and European networks the resulting decision graphs are sufficiently small to be presented to the user. They consist of 8 stops and 19 arcs on average (Germany), roughly doubling on Europe. However, for London these figures are impractically large, increasing to 2724 (stops) and 30243 (arcs). Note that in a dense metropolitan network (such as London), trips operate much more frequently, therefore, many more alternate (and fall-back) journeys exist. These must all be captured by the output. Regarding query time, pCSA-P computes solutions in under $205 \mathrm{~ms}$ on Germany and Europe for all scenarios. On London, all-to-one queries take $668 \mathrm{~ms}$, whereas one-to-one queries can be computed in $272 \mathrm{~ms}$ time. Note that all values are still practical for interactive scenarios.

\section{$7 \quad$ Final Remarks}

In this work, we introduced the Connection Scan framework of algorithms (CSA) for several public transit route planning problems. One of its strengths is the conceptual simplicity, allowing easy implementations. Yet, it is sufficiently flexible 
to handle complex scenarios, such as multi-criteria profile queries. Moreover, we introduced the MEAT problem which considers stochastic delays and asks for a robust set of journeys minimizing (in its entirety) the user's expected arrival time. We extended CSA to MEAT queries in a sound manner. Our experiments on the metropolitan network of London revealed that CSA is faster than existing approaches, and computes solutions to the MEAT problem surprisingly fast in $272 \mathrm{~ms}$ time. All scenarios considered are fast enough for interactive applications. For future work, we are interested in investigating network decomposition techniques to make CSA more scalable, as well as more realistic delay models. Also, since CSA does not use a priority queue, parallel extensions seem promising. Regarding multimodal scenarios, we like to combine CSA with existing techniques developed for road networks.

\section{References}

1. H. Bast. Car or Public Transport - Two Worlds. In S. Albers, H. Alt, and S. Näher, editors, Efficient Algorithms, LNCS 5760, pp. 355-367. Springer, 2009.

2. H. Bast, E. Carlsson, A. Eigenwillig, R. Geisberger, C. Harrelson, V. Raychev, and F. Viger. Fast Routing in Very Large Public Transportation Networks using Transfer Patterns. In ESA, LNCS 6346, pp. 290-301. Springer, 2010.

3. R. Bauer, D. Delling, and D. Wagner. Experimental Study on Speed-Up Techniques for Timetable Information Systems. Networks, 57(1):38-52, 2011.

4. A. Berger, D. Delling, A. Gebhardt, and M. Müller-Hannemann. Accelerating Time-Dependent Multi-Criteria Timetable Information is Harder Than Expected. In $A T M O S$, OpenAccess Series in Informatics (OASIcs), 2009.

5. A. Berger, A. Gebhardt, M. Müller-Hannemann, and M. Ostrowski. Stochastic Delay Prediction in Large Train Networks. In ATMOS, pp. 100-111. 2011.

6. D. Delling, B. Katz, and T. Pajor. Parallel Computation of Best Connections in Public Transportation Networks. ACM JEA, 2012. To appear.

7. D. Delling, T. Pajor, and R. F. Werneck. Round-Based Public Transit Routing. In ALENEX, pp. 130-140. SIAM, 2012.

8. D. Delling, P. Sanders, D. Schultes, and D. Wagner. Engineering Route Planning Algorithms. In Algorithmics of Large and Complex Networks, LNCS 5515, pp. 117-139. Springer, 2009.

9. Y. Disser, M. Müller-Hannemann, and M. Schnee. Multi-Criteria Shortest Paths in Time-Dependent Train Networks. In WEA, LNCS 5038, 347-361. Springer, 2008.

10. R. Geisberger. Contraction of Timetable Networks with Realistic Transfers. In $S E A$, LNCS 6049. Springer, May 2010.

11. M. Goerigk, M. Knoth, M. Müller-Hannemann, M. Schmidt, and A. Schöbel. The Price of Robustness in Timetable Information. In ATMOS, pp. 76-87. 2011.

12. HaCon website, 2013. http://www.hacon.de/hafas/.

13. London Data Store. http://data.london.gov.uk.

14. M. Müller-Hannemann, F. Schulz, D. Wagner, and C. Zaroliagis. Timetable Information: Models and Algorithms. In Algorithmic Methods for Railway Optimization, LNCS 4359, pp. 67-90. Springer, 2007.

15. E. Pyrga, F. Schulz, D. Wagner, and C. Zaroliagis. Efficient Models for Timetable Information in Public Transportation Systems. ACM JEA, 12(2.4):1-39, 2008.

16. C. Sommer. Shortest-Path Queries in Static Networks, 2012. Submitted. Preprint available at http://www.sommer.jp/spq-survey.htm. 